\title{
MicroRNA-10b promotes migration and invasion through Hoxd10 in human gastric cancer
}

Yuan-Yu Wang ${ }^{1 *}$, Li Li ${ }^{2}$, Zai-Yuan Ye ${ }^{1}$, Zhong-Sheng Zhao ${ }^{3 *}$ and Zhi-Long Yan ${ }^{1}$

\begin{abstract}
Background: This study aims to investigate the effect of miR-10b overexpression on cancer cell proliferation, migration, invasion, and Hoxd10 expression.

Methods: The effect of miR-10b on proliferation, migration, and invasion of MKN-28, BGC-823, and SGC-7901 cells and the expression of Hoxd10 protein in SGC-7901 and BGC-823 cells were detected following transfection of miR-10b inhibitor or Negative Control B. Expression of Hoxd10 protein in 436 paraffin-embedded cancer tissues was also investigated.

Results: miR-10b was significantly upregulated in AGS, MKN-28, BGC-823, HCG-27, SGC-7901, and MKN-45 cell lines, miR-10b inhibitor significantly inhibited proliferation and migration of MKN-45, BGC-823 and SGC-7901 cells $48 \mathrm{~h}$ after transfection, while Hoxd10 protein in these cells lines had increased $72 \mathrm{~h}$ after transfection. Hoxd10 was highly expressed in gastric cancer and correlated with size of tumor, Lauren classification, depth of invasion, lymph node and distant metastasis, Tumor-Node-Metastasis (TNM) stage, and prognosis.
\end{abstract}

Conclusions: miR-10b promotes migration and invasion through Hoxd10 in human gastric cancer cell lines and may play an important role in tumorigenesis, progression, and prognosis.

Keywords: Gastric cancer, miR-10b, Hoxd10, Proliferation, Migration, Invasion

\section{Background}

MicroRNAs (miRNAs) are a family of 21-to 25-nucleotide, noncoding small RNAs that primarily function as gene regulators by regulating mRNA translation and degradation [1]. miRNAs play a role in cancer or cancer prevention by adjusting the expression of downstream mRNA [2]. Previous studies show that miR-10b is upregulated in liver, pancreatic, and esophageal cancer, breast cancer with distant metastasis, and glioma tissues, and its expression is closely related with tumor progression [3-7]. miR-10b promotes migration and invasion through HOXD10, CADM1, MAPRE1, and KLF4 et al. [5-10], but the exact regulatory pathway is still poorly understood. However, nothing is known about the role of miR-10b gastric cancer metastasis. Therefore, in this study, we analyzed the level of miR-10b in human gastric cancer cell lines AGS, MKN-28, BGC-

\footnotetext{
*Correspondence: lywyy1979@126.Com; zhaozhongsheng1950@163.com 'Departments of Gastrointestinal Surgery and Pathology, Zhejiang Provincial People's Hospital, Hangzhou 310014, People's Republic of China

${ }^{3}$ Departments of Pathology, Zhejiang Provincial People's Hospital, Hangzhou 310014, Zhejiang, People's Republic of China

Full list of author information is available at the end of the article
}

823, HCG-27, SGC-7901, 9811P, and MKN-45 and in the non-malignant gastric epithelial cell line GES-1, and the effect of miR-10b expression on gastric cancer cell proliferation, invasion, and migration. We aimed to elucidate the role of miR-10b in gastric cancer formation, development, invasion and metastasis, and its mechanism.

\section{Methods \\ Cell culture}

Human gastric cancer cell lines AGS, MKN-28, BGC-823, HCG-27, SGC-7901, 9811P, and MKN-45 and nonmalignant gastric epithelial cell line GES-1 were obtained from Key Laboratory of Gastroenterology of Zhejiang Province (Hangzhou, China) and cultured in RPMI1640 containing $10 \%$ fetal bovine serum (FBS), $50 \mathrm{U} / \mathrm{ml}$ penicillin, and $50 \mu \mathrm{g} / \mathrm{ml}$ streptomycin. All cells were maintained at $37^{\circ} \mathrm{C}$ under an atmosphere of $5 \% \mathrm{CO}_{2}$.

\section{Archived gastric tissue samples and non-tumor mucosa Gastric cancer tissues were collected from gastrectomy specimens from 436 patients (311 male, 125 female,}


median age 60.0 years, range 30-91) from the Department of Surgery, Zhejiang Provincial People's Hospital, from January 1998 to January 2004. Tissues were formalinfixed, paraffin-embedded, and diagnosed clinically and histopathologically at the Departments of Gastrointestinal Surgery and Pathology. The follow-up deadline was December 2008. The survival time was calculated from the date of surgery to the follow-up deadline or date of death, which was mainly due to carcinoma recurrence or metastasis. There were 55, 163, and 218 cases from the cardia, body, and antrum, respectively. According to the 2002 WHO histological classification of gastric carcinoma, there were 326 tubular adenocarcinomas, 16 papillary adenocarcinomas, 29 mucinous adenocarcinomas, and 65 signet-ring cell carcinomas. Thirteen were classified as highly differentiated adenocarcinomas, 128 as well or moderately differentiated adenocarcinomas, 293 as poorly differentiated and 2 as undifferentiated adenocarcinomas. There were 61 cases with distant metastasis. Ninety cases were categorized as stage I, 104 as stage II, 173 as stage III, and 69 as stage IV. Ninety-two noncancerous human gastric tissues were obtained from gastrectomies of adjacent gastric cancer margins greater than $5 \mathrm{~cm}$. Routine chemotherapy was given to the patients with advanced stage disease after surgery, but no radiation treatment was administered to any patients included in our study.

\section{RT-PCR quantification of miR-10b}

RT-PCR was performed to determine the expression of miR-10b. Briefly, total RNA was extracted from human gastric cancer cell lines AGS, MKN-28, BGC-823, HCG27, SGC-7901, 9811P, MKN-45 and non-malignant gastric epithelial cell line GES-1 using Trizol (Invitrogen) according to the manufacturer's instructions. cDNA synthesis was carried out with the miScript Reverse Transcription Kit (Qiagen). The specific reverse primer for miR-10b and U6 were provided by Qiagen. The resulting cDNA was amplified with the QuantiTect SYBR Green PCR Master Mix (Qiagen) using ABI 7500 FAST Realtime PCR (Applied Biosystems). PCR parameters were as follows: $95{ }^{\circ} \mathrm{C}$ for $15 \mathrm{~min}$, followed by 40 cycles of $94{ }^{\circ} \mathrm{C}$ for $15 \mathrm{~s}, 55{ }^{\circ} \mathrm{C}$ for $30 \mathrm{~s}, 72{ }^{\circ} \mathrm{C}$ for 34 s. At the end of the PCR cycles, melting curve analysis was performed. The expression of miR-10b in cancer tissues was compared to matched normal samples using the $2^{-\triangle \Delta C T}$ method, and the expression of miR-10b in gastric cancer cells was compared to GES-1.

\section{miR-10b transfection}

miR-10b inhibitor (miRCURY LNA ${ }^{\mathrm{mm}}$ microRNA Power inhibitor, $5 \mathrm{nmol}, 5^{\prime}$-fluorescein labeled, Exiqon) and Negative Control B (miRCURY LNA ${ }^{\text {tix }}$ microRNA Power Inhibitor Negative Control B, $5 \mathrm{nmol}, 5^{\prime}$-fluorescein labeled, Exiqon) were transfected into MKN-28, BGC-823, and SGC-7901 cells grown in six-well dishes (plated at $5.0 \times 10^{5}$ cells per well $24 \mathrm{~h}$ before transfection). Transfection was performed with Lipofectamine 2000 (Invitrogen). Twenty-four hours after, transfection cells were assayed for migration, invasion, and proliferation.

\section{Cell proliferation assay}

The effect of miR-10b on proliferation of gastric cancer cells was evaluated by MTT assays. MKN-28, BGC-823, and SGC-7901 cells were seeded into 96-well plates at a density of $6.0 \times 10^{3}$ cells/well in quintuplicate and allowed to adhere overnight. Ten microliters of MTT $(5 \mathrm{mg} / \mathrm{ml}$ ) (Sigma) was added to each well 48 or $72 \mathrm{~h}$ after infection, and cells were incubated for a further $4 \mathrm{~h}$. Media was then removed, and $150 \mu \mathrm{l}$ DMSO was added. Absorbance (A) at 570 and $630 \mathrm{~nm}$ were measured using a microplate reader. Relative cell proliferation (\%) was shown by the following equation: Relative proliferation rate $(\%)=\left(\mathrm{A}_{570 \mathrm{~nm}}-\mathrm{A}_{630 \mathrm{~nm}}\right)$ of study group $/$ $\left(\mathrm{A}_{570 \mathrm{~nm}}-\mathrm{A}_{630 \mathrm{~nm}}\right)$ of control group $\times 100 \%$.

\section{In vitro cell migration and invasion assays}

Twenty-four hours after infection, cells were harvested and subjected to the following assays. For migration assays, infected cells $(6 \times 104)$ were plated in triplicate in the top chamber of Transwells (Millicell Hanging Cell Culture Inserts, PIEP12R48, Millipore Corporation) with a membrane containing $8-\mu \mathrm{m}$ diameter pores in $300 \mu \mathrm{l}$ serum-free RPMI1640. The inserts were then placed into the bottom chamber wells of a 24-well plate containing RPMI1640 with $20 \%$ FBS as a chemo-attractant. After $24 \mathrm{~h}$ of incubation, cells remaining on the insert top layer were removed by cotton swab scrubbing, and cells on the lower surface of the membrane were fixed in $100 \%$ methanol for 15 min, followed by staining with Giemsa solution. Cell numbers in five random fields $(400 \times)$ were counted for each chamber and the average values were calculated.

For invasion assays, infected cells $(1.5 \times 105)$ were plated in the top chamber with Matrigel-coated membrane (QCM ECMatrix Cell Invasion Assay, Millipore Corporation), whereas the bottom chambers were filled with conditioned medium. After $48 \mathrm{~h}$ incubation, migrated cells (lower side of the membrane) were counted as described above.

\section{Immunoblotting}

Cells were lysed in RIPA buffer $(150 \mathrm{mM} \mathrm{NaCl}, 10 \mathrm{mM}$ Tris, pH 7.5, 1 \% NP40, 1 \% deoxycholate, 0.1 \% SDS, protease inhibitor cocktail (Roche)). Total proteins were fractionated using the NuPAGE 4-12\% Bis-Tris gradient gel (Invitrogen) and transferred onto PVDF membrane. Membranes were blocked with $5 \%$ non-fat milk in PBS/ 


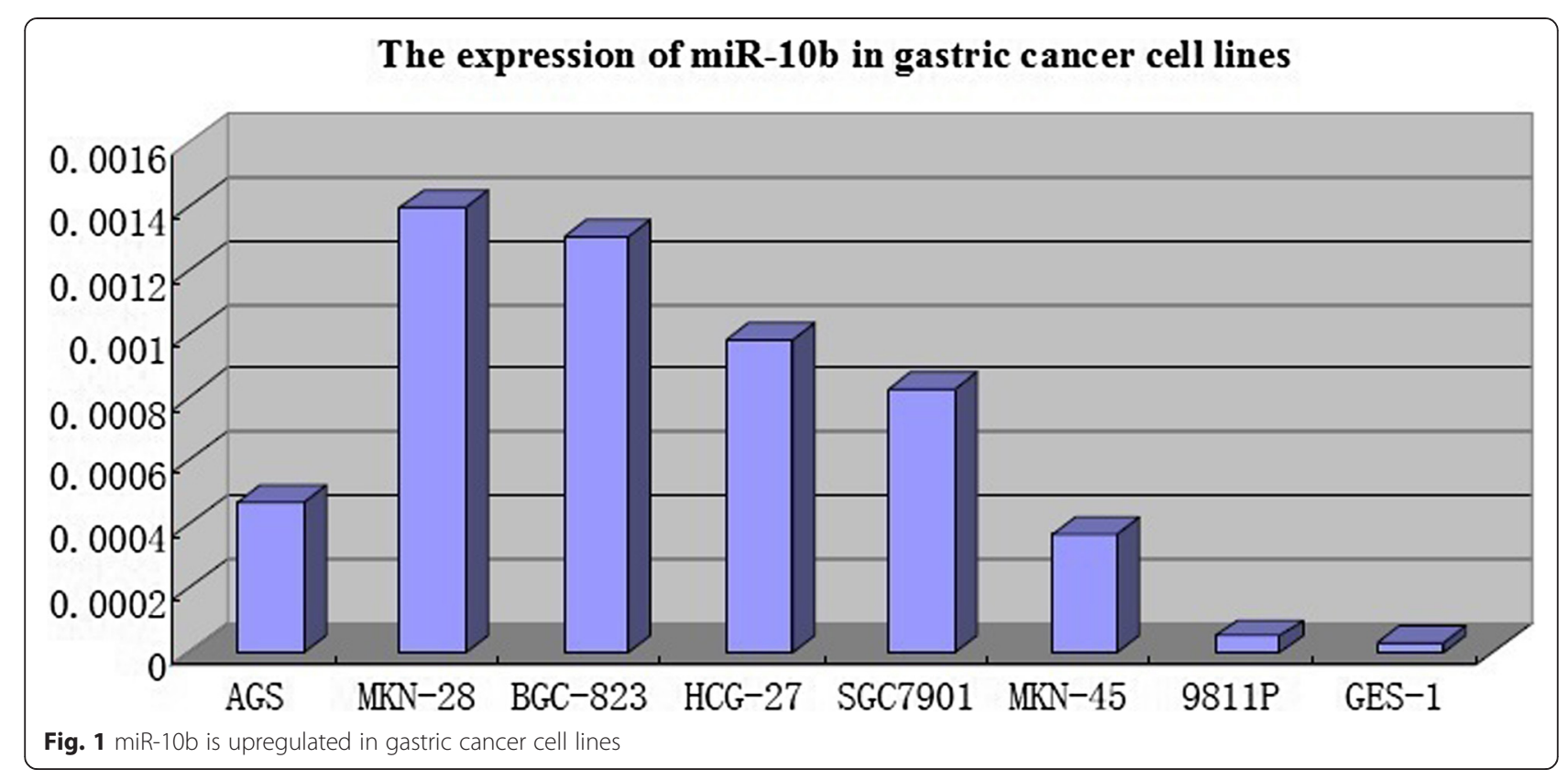

Tween-20 and incubated with antibodies against Hoxd10 (1:200, Santa Cruz) and $\beta$-actin (1:10,000, Abcam).

\section{Immunohistochemistry}

Immunohistochemical analysis was performed as previously described [10]. Sections were incubated with mouse anti-Hoxd10 (1:50; Santa Cruz) overnight at $4{ }^{\circ} \mathrm{C}$. The degree of immunostaining was reviewed and scored independently by two observers based on the proportion of positively stained tumor cells and intensity of staining. Tumor cell proportion was scored as follows: $0(\leq 5 \%$ positive tumor cells), 1 (6-25\% positive tumor cells), 2 (26-50\% positive tumor cells), and 3 (>51\% positive tumor cells). Staining intensity was graded according to the following criteria: 0 (no staining), 1 (weak staining = light yellow), 2 (moderate staining = yellow brown), and 3 (strong staining=brown). Staining index was calculated as the product of staining intensity score and the proportion of positive tumor cells. Using this method of assessment, we evaluated Hoxd10 expression in benign

Table 1 The number of migratory cells after infected with miR-10b inhibitor

\begin{tabular}{llll}
\hline & MKN-28 & BGC-823 & SGC-7901 \\
\hline miR-10b inhibitor & $48.8 \pm 3.96$ & $54.2 \pm 3.19$ & $35.4 \pm 2.07$ \\
Negative Control B & $98.8 \pm 2.59$ & $77.4 \pm 2.07$ & $56.2 \pm 2.77$ \\
Normal Control & $107.4 \pm 5.27$ & $79.4 \pm 3.65$ & $60.2 \pm 3.35$ \\
$t^{\text {t直 }^{\mathrm{a}}}$ & 23.62 & 13.62 & 13.43 \\
t值 $^{\mathrm{b}}$ & 19.87 & 11.62 & 14.09 \\
P值 & 0.0001 & 0.0001 & 0.0001 \\
\hline
\end{tabular}

${ }^{a}$ miR-10b inhibitor vs Negative Control B

${ }^{b} \mathrm{miR}-10 \mathrm{~b}$ inhibitor vs Normal Control gastric epithelia and malignant lesions by determining the staining index with scores of $0,1,2,3,4,6$, or 9 . The cutoff value for high and low expression level was chosen based on a measure of heterogeneity using the log-rank test with respect to overall survival. An optimal cutoff value was identified: a staining index score of $\geq 4$ was used to define tumors with high Hoxd10 expression and a staining index score of $\leq 3$ was used to indicate low Hoxd10 expression.

\section{Statistical analysis}

All statistical analyses were performed using SPSS 16.0 software. Data were analyzed using Student's $t$ test, while categorical data were studied using the $\chi^{2}$ or Fisher exact test. Survival curves were estimated using the KaplanMeier method, and the log-rank test was used to

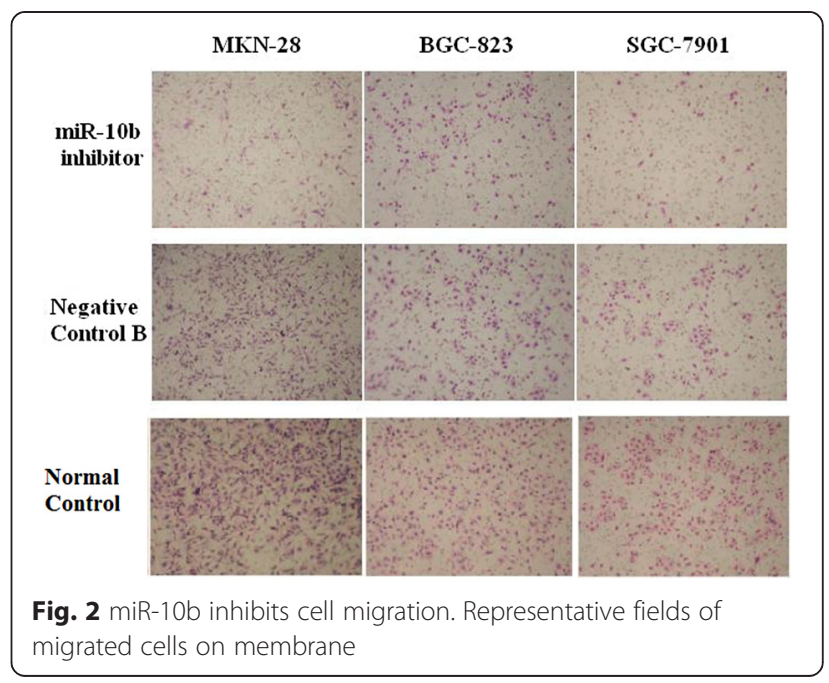




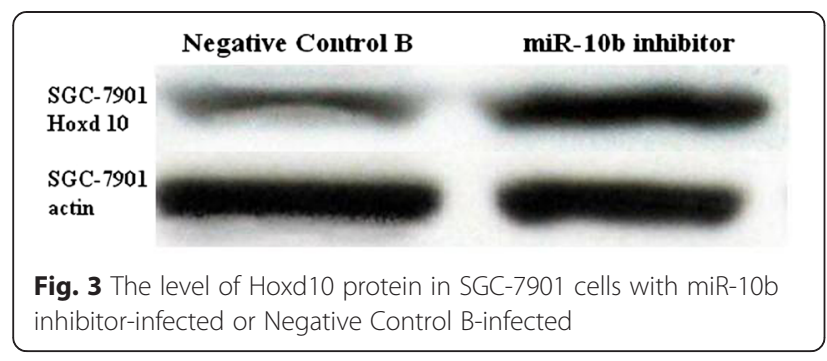

calculate differences between the curves. Multivariate analysis using the Cox proportional hazards regression model was performed to assess the prognostic values of protein expression. Correlation coefficients between protein expression and clinicopathological findings were estimated using the Pearson correlation method. Statistical significance was set at $P<0.05$.

\section{Results}

miR-10b is upregulated in gastric cancer cell lines miR-10b was significantly upregulated in AGS, MKN-28, BGC-823, HCG-27, SGC-7901, and MKN-45 cell lines $\left(4.7 \times 10^{-4}, 1.4 \times 10^{-3}, 1.3 \times 10^{-3}, 9.8 \times 10^{-4}, 8.3 \times 10^{-4}\right.$, and $3.7 \times 10^{-4}$, respectively) compared with the nonmalignant gastric epithelial cell line GES-1 $\left(3.2 \times 10^{-5}\right)$ $(P<0.05)$. The expression of miR-10b did not significantly differ between $9811 \mathrm{P}$ cells $\left(4.99 \times 10^{-5}\right)$ and nonmalignant GES-1 cells $\left(3.2 \times 10^{-5}\right)(P>0.05)($ Fig. 1$)$.

\section{miR-10b inhibited cell proliferation, migration, and invasion in vitro}

To study the biological role of miR-10b in gastric cancer, miR-10b inhibitor or Negative Control B was transfected into MKN-28, BGC-823, and SGC-7901 cells, and miR$10 \mathrm{~b}$ levels were evaluated by RT-PCR. miR-10b levels in SGC-7901, BGC-823, and MKN-28 cells transfected with miR-10b inhibitor were much lower than in Negative Control B-infected cells $(P<0.05), 24 \mathrm{~h}$ after transfection.

Then, we evaluated the effect of miR-10b on the proliferation of gastric cancer cell lines using MTT assays. miR-10b inhibitor significantly inhibited proliferation of MKN-45, BGC-823, and SGC-7901 cells $48 \mathrm{~h}$ after transfection $(P<0.05)$. miR-10b inhibitor significantly inhibited proliferation of BGC-823 cells $72 \mathrm{~h}$ after

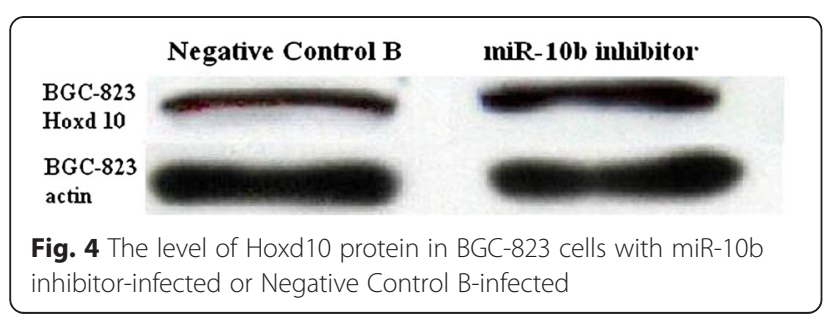

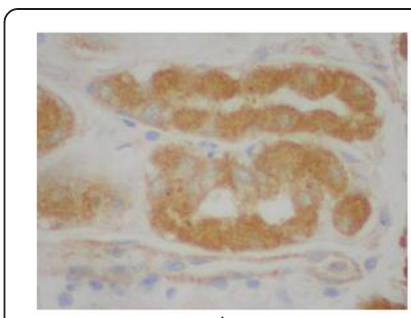

A

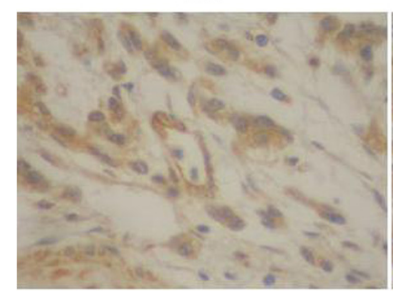

C

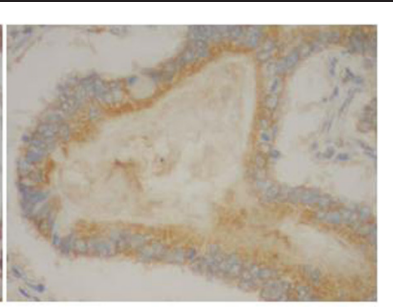

B

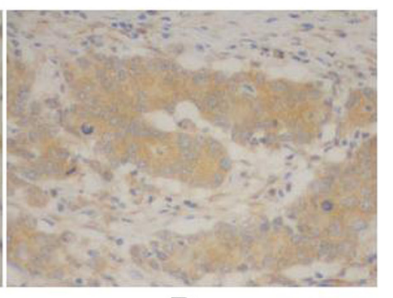

$\mathrm{D}$
Fig. 5 Immunohistochemical staining for Hoxd10 in gastric cancer lesions and noncancerous tissues, magnification 400x. a Hoxd10 was highly expressed in noncancerous tissues. $\mathbf{b}$ Hoxd10 was highly expressed in tubular adenocarcinoma. c Hoxd10 was highly expressed in poorly differentiated adenocarcinoma. $\mathbf{d}$ Hoxd10 was highly expressed in poorly differentiated adenocarcinoma.

transfection $(P<0.05)$ but only had a slight effect on the proliferation of MKN-45 and SGC-7901 cells $(P>0.05)$.

We further analyzed the effect of miR-10b on the migratory and invasive behavior of gastric cell lines (Table 1, Fig. 2). Gastric cancer cells infected with miR$10 \mathrm{~b}$ inhibitor displayed significantly lower transmembrane migration capacity compared with those infected with Negative Control B cells and Normal Control cells $48 \mathrm{~h}$ after transfection $(P<0.05)$. No gastric cancer cell lines transfected with miR-10b inhibitor and Negative Control B invaded through the membrane of QCM ECMatrix Cell Invasion Assay $72 \mathrm{~h}$ after transfection.

\section{miR-10b directly regulated Hoxd10}

To understand the mechanisms by which miR-10b induces tumor invasion and metastasis, Western blotting for Hoxd10 in SGC-7901 and BGC-823 cells was performed at $72 \mathrm{~h}$ post-transfection. Hoxd10 expression was much higher in cells transfected with miR-10b inhibitor than in Negative Control B-infected cells (Figs. 3 and 4).

\section{Expression of Hoxd10 in gastric cancer and clinicopathological features and prognosis}

Hoxd10 was upregulated in $82(89.13 \%)$ normal gastric mucosa samples (Fig. 5), and in 242 of 436 (55.5\%) cases of gastric cancer (Fig. 5). High expression of Hoxd10 correlated with size of tumor, Lauren classification, depth of invasion, lymph node and distant metastasis, and TNM stage (Table 2). We also analyzed the relationship between Hoxd10 expression and prognosis. 
Table 2 Relationship of Hoxd10 expression with pathological parameters of tumor

\begin{tabular}{|c|c|c|c|c|}
\hline \multirow[t]{2}{*}{ Clinical parameters } & \multicolumn{4}{|l|}{ HOXD10 } \\
\hline & Low & High & $t / x^{2}$ & $P$ \\
\hline Age (years) & $59.77 \pm 12.44$ & $58.48 \pm 11.85$ & 1.107 & 0.269 \\
\hline Gender & & & 0.519 & 0.4771 \\
\hline Male & 135 (43.4 \%) & $176(56.6 \%)$ & & \\
\hline Female & 59 (47.2 \%) & $66(52.8 \%)$ & & \\
\hline Location & & & 4.379 & 0.112 \\
\hline Proximal & 31 (56.4 \%) & $24(43.6 \%)$ & & \\
\hline Middle & 74 (45.4 \%) & 89 (54.6\%) & & \\
\hline Distal & 89 (40.8 \%) & $129(59.2 \%)$ & & \\
\hline Size & & & 25.716 & 0.0001 \\
\hline$<5 \mathrm{~cm}$ & $88(34.4 \%)$ & $168(65.6 \%)$ & & \\
\hline$\geq 5 \mathrm{~cm}$ & 106 (58.9 \%) & 74 (41.1\%) & & \\
\hline Lauren classification & & & 63.165 & 0.0001 \\
\hline Intestinal & 58 (26.0 \%) & 165 (74.0 \%) & & \\
\hline Diffuse & 136 (63.8 \%) & 77 (36.2 \%) & & \\
\hline Histology classification & & & 7.343 & 0.062 \\
\hline Papillary adenocarcinoma & $3(18.8 \%)$ & $13(81.2 \%)$ & & \\
\hline Tubular adenocarcinoma & 142 (43.6 \%) & $184(56.4 \%)$ & & \\
\hline Mucinous adenocarcinoma & 17 (58.6 \%) & $12(41.4 \%)$ & & \\
\hline Signet-ring cell carcinoma & 32 (49.2 \%) & $33(50.8 \%)$ & & \\
\hline Histologic differentiation & & & 5.25 & 0.154 \\
\hline Well & $3(23.1 \%)$ & 10 (76.9\%) & & \\
\hline Moderately & 50 (39.1\%) & 78 (60.9 \%) & & \\
\hline Poorly & 140 (47.8 \%) & 153 (52.2 \%) & & \\
\hline Others & $1(50.0 \%)$ & $1(50.0 \%)$ & & \\
\hline Invasion depth & & & 33.892 & 0.0001 \\
\hline $\mathrm{T} 1$ & $9(15.8 \%)$ & 48 (84.2 \%) & & \\
\hline $\mathrm{T} 2$ & 39 (35.8 \%) & $70(64.2 \%)$ & & \\
\hline T3 & 129 (52.9 \%) & 115 (47.1\%) & & \\
\hline $\mathrm{T} 4$ & 17 (65.4 \%) & $9(34.6 \%)$ & & \\
\hline TNM stages & & & 60.859 & 0.0001 \\
\hline I & 17 (18.9 \%) & $73(81.1 \%)$ & & \\
\hline$\|$ & 30 (28.8 \%) & 74 (71.2 \%) & & \\
\hline III & 102 (59.0 \%) & 71 (41.0\%) & & \\
\hline IV & 45 (65.2 \%) & $24(34.8 \%)$ & & \\
\hline Lymphatic metastasis & & & 45.165 & 0.0001 \\
\hline No & 40 (24.1\%) & 126 (75.9\%) & & \\
\hline Yes & $154(57.0 \%)$ & $116(43.0 \%)$ & & \\
\hline Regional lymph nodes & & & 51.95 & 0.0001 \\
\hline PNO & 40 (24.1\%) & 126 (75.9 \%) & & \\
\hline PN1 & 67 (49.3\%) & 69 (50.7\%) & & \\
\hline PN2 & 65 (65.7\%) & $34(34.3 \%)$ & & \\
\hline PN3 & 22 (62.9\%) & $13(37.1 \%)$ & & \\
\hline
\end{tabular}


Table 2 Relationship of Hoxd10 expression with pathological parameters of tumor (Continued)

\begin{tabular}{llll}
\hline Distant metastasis & & 14.821 & 0.0001 \\
No & $153(40.8 \%)$ & $222(59.2 \%)$ & \\
Yes & $41(67.2 \%)$ & $20(32.8 \%)$ & \\
\hline
\end{tabular}

The cumulative 5-year survival rate was $53.7 \%$ in the high Hoxd10 protein expression group but was only $24.7 \%$ in the low expression group $\left(\chi^{2}=60.81, P=\right.$ 0.001) (Fig. 6). In stages I, II, and III, the 5-year survival rate of patients with high expression of Hoxd10 was significantly higher than those in patients with low expression. In stage I, the cumulative 5-year survival rate was $91.8 \%$ in the high Hoxd10 expression group but was only $82.4 \%$ in low expression group $\left(\chi^{2}=4.25, P=\right.$ 0.039) (Fig. 7). In stage II, the cumulative 5-year survival rate was $68.9 \%$ in high Hoxd10 expression group but was only $53.3 \%$ in low expression group $\left(\chi^{2}=5.35, P=\right.$ 0.021) (Fig. 8). In stage III, the cumulative 5-year survival rate was $40.8 \%$ in high Hoxd10 expression group but was only $15.7 \%$ in low expression group $\left(\chi^{2}=12.87\right.$, $P=0.0001$ ) (Fig. 9). In stage IV, the expression of Hoxd10 did not correlate with the 5-year survival rate, the cumulative 5-year survival rate was $4.5 \%$ in high Hoxd10 expression group but was only $2.3 \%$ in low expression group in stage IV $\left(\chi^{2}=2.19, P=0.138\right)$ (Fig. 10). The factors with possible prognostic effects in gastric carcinoma were analyzed by Cox regression analysis. The study revealed that lymph node $(P=0.012)$ and distant metastases $(P=0.008)$, TNM stage $(P=0.001)$, and expression of Hoxd10 $(P=0.008)$ were independent prognostic factors in patients with gastric carcinoma.
However, age, sex, tumor location and size, histological classification, tumor differentiation, Lauren's classification, and regional lymph node stage had no prognostic value.

\section{Discussion}

Recent evidence has shown that altered patterns of microRNA (miRNA) expression correlate with various human cancers. miR-10b was recently reported to be dysregulated in some types of cancer and to play a role in invasion and metastasis. Single microRNA (miRNA) can regulate expression of several or multiple principal targets in a specific microenvironment. In different cellular contexts, the same miRNA may exhibit diverse functions, depending on the repertoire and stoichiometry of its direct mRNA targets [11]. For instance, in breast cancer, microRNA-10b (miR-10b) promotes invasion and metastasis of tumor cells through regulation of HOXD10, E-cadherin, and syndecan-1 [12, 13]. miR-10b may positively regulate the invasion and metastasis of hepatocellular carcinoma through targeting CADM [8]. miR-10b induces glioma cell invasion by modulating MMP-14 and UPAR expression via HOXD10 [14].

The oncogenic or tumor suppressive effects of miR10b might be tissue specific. Some debate exists on whether miR-10b is upregulated or downregulated in

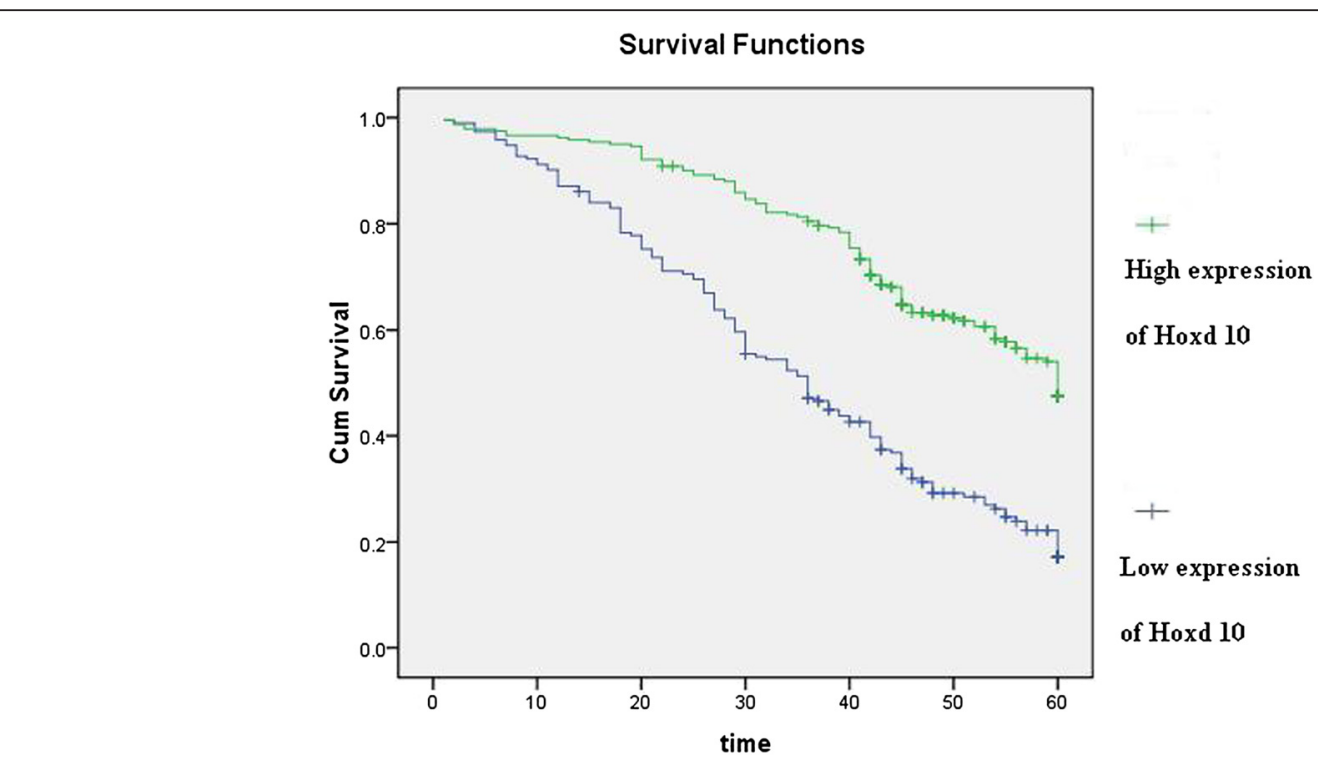

Fig. 6 Kaplan-Meier curves with univariate analyses (log-rank) for patients with simultaneously low Hoxd10 expression versus high Hoxd10 expression tumors in all gastric cancer 


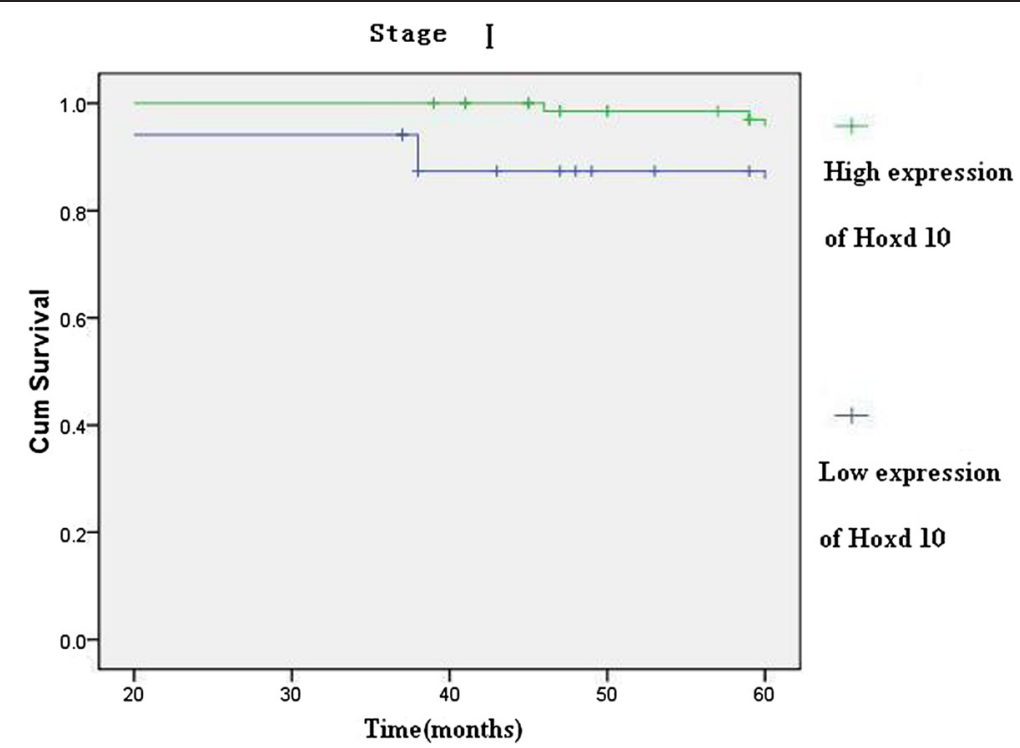

Fig. 7 Kaplan-Meier curves with univariate analyses (log-rank) for patients with low Hoxd10 expression versus high Hoxd10 expression tumors in all gastric cancer in stage I

gastric cancer $[9,15]$. Our previous study found miR$10 \mathrm{~b}$ to be significantly upregulated in tissues, overexpression of miR-10b in gastric cancer tissues was associated with lymph node and distant metastases, and the results suggest that miR-10b may play an important role in gastric tumorigenesis, progression, and prognosis [16]. However, effects and potential mechanisms of action of miR-10b in the metastasis of gastric cancer have not been explored thoroughly. Here, we discuss a unique role of miR-10b in gastric cancer cell invasion and metastasis. In this study, we analyzed the level of miR-10b in human gastric cancer cell lines AGS, MKN-28, BGC823, HCG-27, SGC-7901, 9811P, and MKN-45 and in the non-malignant gastric epithelial cell line GES-1, and

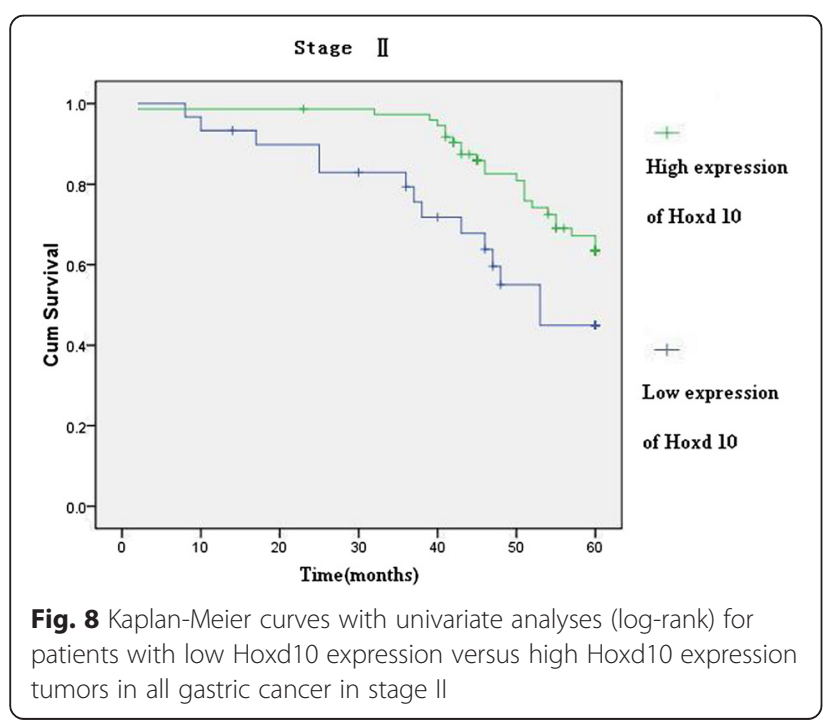

the effect of miR-10b expression on gastric cancer cell proliferation, invasion, and migration. miR-10b was significantly upregulated in AGS, MKN-28, BGC-823, HCG-27, SGC-7901, and MKN-45 cell lines compared with the non-malignant gastric epithelial cell line GES-1. Liu et al. found miR-10b was highly expressed in poorly differentiated gastric cancer cell lines, such as BGC-823 and MKN45 [9]. We further analyzed the effect of miR$10 \mathrm{~b}$ on the migratory and invasive behavior of gastric cell lines (BGC-823, SGC-7901, and MKN-45). miR-10b inhibitor significantly inhibited proliferation and migration and also significantly increased the level of Hoxd10 protein in SGC-7901 and BGC-823 cells. Some debate exists on whether miR-10b is inhibited or increased

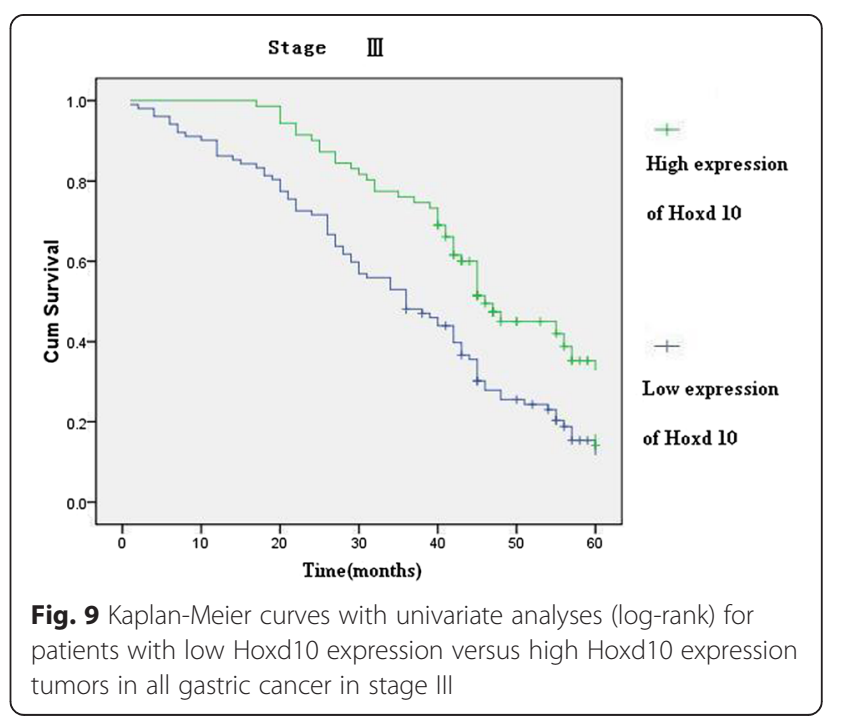




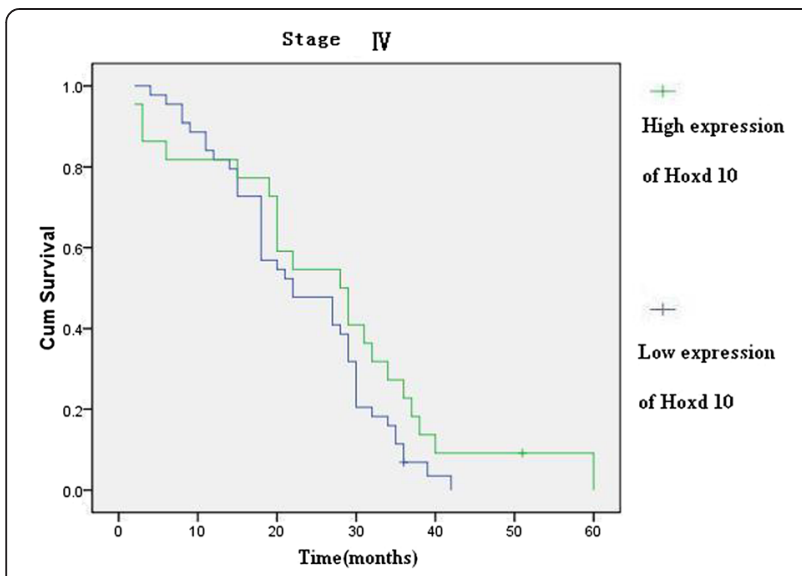

Fig. 10 Kaplan-Meier curves with univariate analyses (log-rank) for patients with low Hoxd10 expression versus high Hoxd10 expression tumors in all gastric cancer in stage IV

migration and invasion in gastric cell lines. miR-10b inhibitor increased both cell migration and invasion [17]. Inhibition of miR-10b activity by miR-10b inhibitor had no obvious effects on migration of MNK45 cells, while severely blocked cell invasion ability [9].

miR-10b can stimulate the upregulation of $\mathrm{RhoC}$ and AKT phosphorylation through targeting HOXD10, thus promoting cell invasion in gastric tumors [9]. There was no literature about the relationship between expression of HOXD10 and prognosis of patients. We further analyzed the association between Hoxd10 expression and clinicopathological features and prognosis. Hoxd10 was overexpressed in gastric cancer and correlated with size of tumor, Lauren classification, depth of invasion, lymph node and distant metastasis, TNM stage, and prognosis.

\section{Conclusions}

In conclusion, we analyzed the expression of miR-10b in gastric cancer cell lines, and the effect of miR-10b on cell proliferation, migration, invasion assays, and Hoxd10 expression. Results reported here increase the understanding of the molecular basis of miR-10b in gastric cancer and suggest that miR-10b promotes migration and invasion through Hoxd10 in human gastric cancer cell lines. miR-10b may be a useful molecular biomarker for assessing the risk of gastric cancer development, and modulation of miR-10b may represent a therapeutic approach for treating gastric cancer.

\footnotetext{
Abbreviations

AKT: $V$-akt murine thymoma viral oncogene homolog 1; CADM1: cell adhesion molecule 1; HOXD10: homeobox D10; KLF4: Kruppel-like factor 4; MAPRE1: microtubule-associated protein, RP/EB family, member 1; MMP14: Matrix metallopeptidase 14; RhoC: Ras homolog family member C; TNM: Tumor-Node-Metastasis; UPAR: plasminogen activator, urokinase receptor.
}

\section{Competing interests}

The authors declare that they have no competing interests.

\section{Authors' contributions}

Y-YW and LL participated in the scientific experiments. All authors participated in the conception and design of the study, as well as data collection and interpretation, manuscript preparation, and literature search. All authors have read and approved the final manuscript.

\section{Acknowledgements}

This work was supported by Zhejiang Provincial Medical Science Research Foundation (201337120) and Zhejiang Provincial Department of Science and Technology Research Foundation (2008C33040).

\section{Author details}

'Departments of Gastrointestinal Surgery and Pathology, Zhejiang Provincial People's Hospital, Hangzhou 310014, People's Republic of China. ${ }^{2}$ Key Laboratory of Gastroenterology of Zhejiang Province, Hangzhou 310014 Zhejiang, People's Republic of China. ${ }^{3}$ Departments of Pathology, Zhejiang Provincial People's Hospital, Hangzhou 310014, Zhejiang, People's Republic of China.

Received: 5 March 2015 Accepted: 5 August 2015

Published online: 28 August 2015

\section{References}

1. Hutvágner G, Zamore PD. A microRNA in a multiple-turnover RNAi enzyme complex. Science. 2002;297(5589):2056-60.

2. Bartels CL, Tsongalis GJ. MicroRNAs: novel biomarkers for human cancer. Clin Chem. 2009;55(4):623-31.

3. Ladeiro Y, Couchy G, Balabaud C, Bioulac-Sage P, Pelletier L, Rebouissou S, et al MicroRNA profiling in hepatocellular tumors is associated with clinical features and oncogene/tumor suppressor gene mutations. Hepatology. 2008;47(6):1955-63.

4. Bloomston M, Frankel WL, Petrocca F, Volinia S, Alder H, Hagan JP, et al. MicroRNA expression patterns to differentiate pancreatic adenocarcinoma from normal pancreas and chronic pancreatitis. JAMA. 2007;297(17):1901-8.

5. Tian Y, Luo A, Cai Y, Su Q, Ding F, Chen H, et al. MicroRNA-10b promotes migration and invasion through KLF4 in human esophageal cancer cell lines. J Biol Chem. 2010;285(11):7986-94.

6. Ma L, Teruya-Feldstein J, Weinberg RA. Tumour invasion and metastasis initiated by microRNA-10b in breast cancer. Nature. 2007;449(7163):682-8.

7. Nakata K, Ohuchida K, Mizumoto K, Kayashima T, Ikenaga N, Sakai H, et al. MicroRNA-10b is overexpressed in pancreatic cancer, promotes its invasiveness, and correlates with a poor prognosis. Surgery. 2011;150(5):916-22.

8. Li QJ, Zhou L, Yang F, Wang GX, Zheng H, Wang DS, et al. MicroRNA-10b promotes migration and invasion through CADM1 in human hepatocellular carcinoma cells. Tumour Biol. 2012;33(5):1455-65.

9. Liu Z, Zhu J, Cao H, Ren H, Fang X. miR-10b promotes cell invasion through RhoC-AKT signaling pathway by targeting HOXD10 in gastric cancer. Int J Oncol. 2012:40(5):1553-60.

10. Zhao ZS, Wang YY, Chu YQ, Ye ZY, Tao HQ. SPARC is associated with gastric cancer progression and poor survival of patients. Clin Cancer Res. 2010;16(1):260-8.

11. Gabriely G, Teplyuk NM, Krichevsky AM. Context effect: microRNA-10b in cancer cell proliferation, spread and death. Autophagy. 2011;7(11):1384-6.

12. Liu Y, Zhao J, Zhang PY, Zhang Y, Sun SY, Yu SY, et al. MicroRNA-10b targets E-cadherin and modulates breast cancer metastasis. Med Sci Monit. 2012;18(8):BR299-308.

13. Ibrahim SA, Yip GW, Stock C, Pan JW, Neubauer C, Poeter M, et al. Targeting of syndecan-1 by microRNA miR-10b promotes breast cancer cell motility and invasiveness via a Rho-GTPase- and E-cadherin-dependent mechanism. Int J Cancer. 2012;131(6):E884-96.

14. Sun L, Yan W, Wang Y, Sun G, Luo H, Zhang J, et al. MicroRNA-10b induces glioma cell invasion by modulating MMP-14 and UPAR expression via HOXD10. Brain Res. 2011;1389:9-18.

15. Kim K, Lee HC, Park JL, Kim M, Kim SY, Noh SM, et al. Epigenetic regulation of microRNA-10b and targeting of oncogenic MAPRE1 in gastric cancer. Epigenetics. 2011;6:740-51.

16. Wang YY, Ye ZY, Zhao ZS, Li L, Wang YX, Tao HQ, et al. Clinicopathologic significance of miR-10b expression in gastric carcinoma. Hum Pathol. 2013:44(7):1278-85.

17. Li Z, Lei H, Luo M, Wang Y, Dong L, Ma Y, et al. DNA methylation downregulated mir-10b acts as a tumor suppressor in gastric cancer. Gastric Cancer. 2015;18(1):43-54 